\title{
Modal filtering for nulling interferometry
}

\section{First single-mode conductive waveguides in the mid-infrared}

\author{
L. Labadie ${ }^{1}$, P. Labeye ${ }^{2}$, P. Kern ${ }^{1}$, I. Schanen ${ }^{3}$, B. Arezki ${ }^{1}$, and J.-E. Broquin ${ }^{3}$ \\ ${ }^{1}$ Laboratoire d'Astrophysique de l'Observatoire de Grenoble, BP 53, 38041 Grenoble Cedex 9, France \\ e-mail: lucas.labadie@obs.ujf-grenoble.fr \\ 2 Laboratoire d'Électronique et des Technologies de l'Information (LETI), CEA-DRT-LETI, 17 rue des Martyrs, \\ 38054 Grenoble Cedex 9, France \\ ${ }^{3}$ Institut de Microélectronique et Photonique, UMR 5130, 23 rue des Martyrs, BP 257, 38016 Grenoble Cedex 1, France
}

Received 11 May 2005 / Accepted 4 November 2005

\section{ABSTRACT}

\begin{abstract}
We have investigated the manufacturing and characterization of first single-mode conductive waveguides to be used as modal filters for nulling interferometry in the mid-infrared range [4-20 $\mu \mathrm{m}]$. As a very high dynamic range is mandatory for the detection of Earth-like planets, modal filtering is a crucial instrumental aspect. The hollow metallic waveguides (HMW) presented here are manufactured using micro-machining techniques. Single-mode behavior has been investigated in the laboratory through polarization analysis and transmission features have been measured using relative flux comparison. Single-mode behavior was assessed at $\lambda=10.6 \mu \mathrm{m}$ for rectangular waveguides with dimensions $a=10 \mu \mathrm{m}$ and $b \leq 5.3 \mu \mathrm{m}$ with an accuracy of $\sim 2.5 \%$. The tests showed that a single-polarization state can be maintained in the waveguide. A comparison with results on multi-mode HMW is given. Excess losses of $2.4 \mathrm{~dB}$ ( $\sim 58 \%$ transmission) have been measured for a singlemode waveguide. In particular, the importance of coupling conditions in the waveguide is emphasized here. The goal of manufacturing and characterizing the first single-mode HMW for the mid-infrared has been achieved. This opens the road to the use of integrated optics for interferometry in this spectral range.
\end{abstract}

Key words. instrumentation: interferometers - methods: laboratory - methods: data analysis

\section{Introduction}

Since the discovery of a Jupiter-mass companion around $51 \mathrm{Peg}$ by Mayor \& Queloz (1995), investigators have searched for habitable telluric planets and indices of life on them. The main challenge is the huge ratio of star to planet flux; thus Bracewell (1978) and Angel et al. (1986) have suggested observing in the mid-infrared range. Indeed, in the case of a Sun/Earth system the luminosity ratio is about $10^{9}$ in the visible while it drops to $10^{6}$ at $10 \mu \mathrm{m}$. The latter authors have shown that reliable biomarkers such as $\mathrm{H}_{2} \mathrm{O}(<8 \mu \mathrm{m}), \mathrm{O}_{3}(9.6 \mu \mathrm{m})$ and $\mathrm{CO}_{2}(15 \mu \mathrm{m})$ could be investigated by spectroscopy in the mid-infrared. At those wavelengths, the search for Earthlike planets would require an infrared nulling interferometer in space operating in the [4-20 $\mu \mathrm{m}$ ] band (Bracewell \& McPhie 1979). A nulling interferometer generates destructive interference for an on-axis star by introducing an achromatic $\pi$ phase shift between the arms of the interferometer, which results in the complete rejection of the starlight. For a well-tuned configuration of the baseline, the signal from an off-axis planet will be transmitted since it interferes constructively at the output of the instrument. Thus, this type of instrument provides both high angular resolution through baselines of several tens of meters and high dynamic range through the intrinsic coronographic feature of its transmission map (Léger et al. 1996).

However, reaching a rejection ratio of $10^{6}$ is subject to three major limitations: phase defects that include residual OPD errors, pointing errors, optics defects and micro-roughness that induce high-frequency defects; Overall and local amplitude shifts; Polarization mismatching due to the rotation of the electric field in the optical train. Concerning the first two points (excepted for residual OPD), some authors have shown that stellar leaks effects could be maintained below $10^{-6}$ by implementing spatial filtering using either pinholes (Ollivier \& Mariotti 1997) or single-mode waveguides (Mennesson et al. 2002). Polarization mismatches could be corrected using polarization maintaining devices.

Filtering with single-mode waveguides is already widely used in near-infrared interferometry. This is done with optical fibers (Coudé du Foresto \& Ridgway 1992) or single-mode integrated optics (IO) (Kern et al. 1997). The latter option provides, in addition to modal filtering, optical functions like beam combination which is integrated on a small optical chip. This solution relaxes instrumental constraints like alignment, 
mechanical and thermal stability (Malbet et al. 1999) and has provided calibrated astrophysical data from several interferometric facilities (Kervella et al. 2003).

The importance of modal filtering is now widely accepted. In the context of mid-infrared nulling interferometry, the lack of single-mode waveguides has resulted in specific studies to develop mid-infrared chalcogenide fibers (Bordé et al. 2003) and silver-halide fibers (Wallner et al. 2004). Wehmeier et al. (2004) provide theoretical studies on conductive waveguides for the mid-infrared.

The present work aims to extend single-mode integrated optics concepts to the mid-infrared range, where it may be advantageous for future space-based interferometers like Darwin (Fridlund 2000) or TPF (Beichman 2001). We present the results on the first single-mode Hollow Metallic Waveguides (HMW) manufactured for the mid-infrared range. HMW is a new approach in the implementation of guided optics properties for mid-infrared interferometry compared to dielectric technologies. Although theory can help us to predict its performance, the novelty of the technology process involves several unknowns such as surface quality, coupling and propagation losses on the final performances. Only first manufacturing and validation tests can provide reliable conclusions to improve the performance in successive iterations.

First, we describe previous quantitative results on the effects of using single-mode waveguides for filtering. Second, we describe the impact of HWM geometry on single-mode behaviour as well as the applied manufacturing process. Then, we discuss the hardware for the characterization phase and the procedure adopted for specific measurements. We present results on the modal behavior and the transmission features of the manufactured HMW and we discuss the results and the hollow metallic waveguide properties.

\section{Modal filtering with single-mode waveguides}

In order to achieve a $10^{5}$ rejection ratio with a four-telescope configuration, Léger et al. (1995) pointed out the following constraints on the instrument:

- Defects due to optical quality and micro-roughness as well as residual OPD should be kept smaller than $\lambda / 2000$ at $10 \mu \mathrm{m}$ (i.e. $\lambda / 100$ in the visible at $0.5 \mu \mathrm{m}$ ).

- Pointing errors should remain below $1 / 80$ of the Airy disk in the visible, or $1 / 1600$ at $10 \mu \mathrm{m}$.

To relax those constraints, Ollivier \& Mariotti (1997) first proposed the use of pinholes to be placed at the focus of each telescope. A pinhole with the size of the Airy disk filters out the small-scale defects (i.e. the high spatial frequencies) providing efficient spatial-cleaning. Defects due to dust and polishing are well corrected with a rejection increase of three orders of magnitude. For instance, pinhole filtering permits us to reach a rejection rate of $10^{6}$ when the transmission is degraded by $1 \%$ due to dust, while it remains below $10^{3}$ without filtering. Pinhole correcting efficiency, however, is reduced for large-scale defects. In particular tilt errors are not significantly corrected with this method and the rejection ratio is improved by less than a factor of two. Also, residual OPD errors cannot be corrected by any type of spatial filtering since they do not affect the intensity distribution of the Airy pattern in the Fourier plane at the telescope focus.

Further improvements are achieved using single-mode waveguides to implement modal filtering. In guided optics theory (Marcuse 1974), a single-mode waveguide propagates only one mode, the fundamental one, whose amplitude spatial distribution is only constrained by the geometrical and optical parameters of the waveguide, independently of the incoming wavefront. Coupling a corrugated wavefront to the fundamental mode converts the input phase fluctuations to overall intensity fluctuations of the propagating mode. The resulting wavefront at the waveguide output is then cleaned of all phase defects. When placed at the focus of each telescope, the fringe visibility is then only affected by amplitude mismatching which is less drastic than phase defects. However, single-mode waveguides provide neither a correction of the residual OPD nor a correction of overall amplitude unbalancing. Those errors can be corrected with classical fringe tracking and active amplitude matching systems.

Mennesson et al. (2002) provided a quantitative comparison of the optical constraints set respectively by no filtering mode, pinhole filtering and single-mode waveguide filtering to reach a typical rejection ratio of $10^{6}$. Concerning highorder optics defects and micro-roughness, the wavefront quality at $\lambda=10 \mu \mathrm{m}$ drops from $\lambda / 4400 \mathrm{rms}$ without spatial filtering to $\lambda / 400 \mathrm{rms}$ with pinhole filters and to $\lambda / 63 \mathrm{rms}$ with a singlemode waveguide, or a gain of almost two order of magnitude. For the telescope pointing errors, single-mode waveguides are very efficient. Reaching a deep null of $10^{6}$ at $10 \mu \mathrm{m}$ requires a control of the tip-tilt up to 1.2 mas rms for $1.5 \mathrm{~m}$-class telescopes. Under the same conditions, modal filtering with singlemode waveguides relaxes this constraint to 38 mas rms. Local amplitude errors due, for instance, to imperfect coating reflectivity can also be corrected more efficiently with single-mode waveguides. For the same previous rejection ratio, the relative local amplitude shift to be monitored goes from $0.2 \%$ without filtering to $2 \%$ with pinhole filtering and to $10 \%$ with singlemode filtering.

A way to remove polarization mismatching is to implement single-mode and single-polarization waveguides in the optical train. This aspect is discussed in Sect. 5.

Modal filtering can be implemented after coherent recombination of the beams using only one waveguide rather than placing a waveguide at each telescope focus. The advantage is that any additional phase corruption after filtering is prevented as are differential effects due to slightly different waveguides.

Modal filtering based on the implementation of singlemode waveguides presents a clear advantage for nulling interferometry where high rejection rates are strongly constrained by the factors presented above. Single-mode waveguides are preferred to pinholes since they are efficient for high-order and low-order defects, while the latter devices are efficient only for small-scale defects. Therefore, developing and manufacturing of single-mode waveguides working in the full band [4-20 $\mu \mathrm{m}$ ] will provide fundamental improvements for nulling interferometry missions like Darwin and TPF. 
Table 1. Description of tested waveguides and experimental performances over $1 \mathrm{~mm}$ length. "-" means the computation is not applicable. The waveguides supporting a taper are referred to as Gxx-T. The modal behavior has been assessed through polarization tests. For $b \leq 5.1 \mu \mathrm{m}$, the transmitted flux can be totally extinguished at $0^{\circ}$ angular position of the polarizer. Above this width, flux variations are still observed but it no longer possible to extinguish the flux. In the single-mode case (SM), the coupling efficiency on the fundamental mode has been computed with the overlap integral method for the different geometrical apertures. In the case of waveguides with tapers (G46-T and G36-T), the coupling efficiencies have been computed for the taper aperture $40 \mu \mathrm{m} \times 10 \mu \mathrm{m}$, which results in similar values of $C_{\text {in }}$ and $C_{\text {out }}$. The excess losses include the propagation losses of the waveguide and the effects of the quality factor $\eta$. Using a taper significantly improves the coupling both in the single-mode and multi-mode case (MM). Multi-mode waveguides with taper transmit about $4.5 \%$ of the total flux while single-mode waveguides with tapers transmit about $2 \%$ of the total flux.

\begin{tabular}{lccccccc}
\hline \hline Reference & $\begin{array}{c}b \\
(\mu \mathrm{m})\end{array}$ & $\begin{array}{c}\text { Modal } \\
\text { behavior }\end{array}$ & $\begin{array}{c}C_{\text {in }} \\
(\%)\end{array}$ & $\begin{array}{c}C_{\text {out }} \\
(\%)\end{array}$ & $\begin{array}{c}T_{\text {exp }} \\
(\%)\end{array}$ & $\begin{array}{c}\text { Excess } \\
\text { losses }(\mathrm{dB})\end{array}$ & Error (dB) \\
\hline G46-1 & 4.7 & SM & 15.5 & 4.3 & 0.26 & $<-4.0$ & 0.13 \\
G46-2 & 4.7 & SM & 15.5 & 4.3 & 0.38 & $<-2.4$ & 0.14 \\
G46-T & 4.7 & SM & 20.9 & 21.3 & 1.9 & $<-3.7$ & 0.13 \\
\hline G36-T & 5.1 & SM & 20.9 & 21.3 & 1.8 & $<-3.9$ & 0.13 \\
\hline G35-T & 7.4 & MM & - & - & 4.9 & - & - \\
\hline G42-T & 9.2 & MM & - & - & 4.2 & - & - \\
\hline G63-1 & 8.4 & MM & - & - & 1.9 & - & - \\
G63-T & 8.4 & MM & - & - & 5.1 & - & - \\
\hline
\end{tabular}

\section{Geometry and manufacturing of the waveguides}

A proposed solution for mid-infrared modal filters are hollow metallic waveguides (HMW), well studied in microwave engineering and widely used in radioastronomy field. Light propagation with HMW is based on multiple metallic reflections of the radiation inside the cavity. HMW are good candidates because they are able to cover the full Darwin band [4-20 $\mu \mathrm{m}$ ] through the implementation of three or four single-mode subbands. Full coverage of the band is similarly addressed when using dielectric solutions since this reduces the number of infrared glassy materials that can be exploited.

The adopted geometry for our devices is rectangular (see Fig. 4) with sides $a$ and $b$ (and $a \geq b$ ). Based on our study of well established microwave models (Rizzi 1988), we have computed the electric field distributions of the different propagated modes as well as their respective cut-off wavelengths (i.e. the wavelength above which the mode is no longer propagated). Thus, for a waveguide geometry fulfilling the condition $a=2 b$, the fundamental mode is the linearly polarized $\mathrm{TE}_{10}$ with a cutoff wavelength $\lambda_{\mathrm{c}\left(\mathrm{TE}_{10}\right)}=2 a$. The first higher-order mode is the mode $\mathrm{TE}_{01}$ with a cut-off wavelength $\lambda_{\mathrm{c}\left(\mathrm{TE}_{01}\right)}=a$ and with $a$ linear polarization orthogonal to the $\mathrm{TE}_{10}$ fundamental mode. Consequently, a waveguide with the specified geometry has a single-mode range defined by those two cut-off wavelengths, that is $a<\lambda<2 a$. Note that the single-mode range remains unchanged with a geometry verifying $b \leq \frac{a}{2}$ while it becomes $2 b<\lambda<2 a$ when $b \geq \frac{a}{2}$. It is then possible to tune the single-mode range of the rectangular waveguide by changing its geometry. Under these conditions, we have fixed for HMW an etching depth of $a=10 \mu \mathrm{m}$ while their width ranges from $b \approx 4.5 \mu \mathrm{m}$ to $b \approx 10 \mu \mathrm{m}$ (see Table 1 ). This geometry theoretically ensures a single-mode behavior at $\lambda=10.6 \mu \mathrm{m}$ for those waveguides that comply with the condition $b \leq 5.3 \mu \mathrm{m}$. The waveguides fulfilling the opposite condition, $b \geq 5.3 \mu \mathrm{m}$, will have a multi-mode behavior at $10.6 \mu \mathrm{m}$. Our work, based on characterization experiments at $10.6 \mu \mathrm{m}$, aims to experimentally enhance the difference in behavior between single-mode type and multi-mode type waveguides.

The manufacturing of a hollow metallic waveguide is based on a standard micro-technology etching process of the silicon substrate plus anodic bonding of a Pyrex cover on the silicon structure. The process is described in Fig. 1. The result is a chip containing rectangular waveguides spaced by $300 \mu \mathrm{m}$ with submicron accuracy, with inside walls coated with gold. The total length of the waveguide is $1 \mathrm{~mm}$. To avoid direct transmission through the silicon substrate, the external facets of the chip are also coated with gold by evaporation.

The results reported in this paper are related to channel waveguides both with and without tapers. A taper is a smooth transition between a multi-mode channel waveguide and a single-mode one, similar to impedance cornets in millimeter astronomy. This structure added at the input and output of the waveguide relaxes the coupling constraints. The dimensions of the taper are $40 \mu \mathrm{m}$ width by $10 \mu \mathrm{m}$ depth (see Fig. 4). The 1-mm length waveguides include the tapers when they are added.

The right panel of Fig. 1 presents a Scanning Electron Microscope (SEM) image of a $10 \times 5 \mu \mathrm{m}$ component. Table 1 summarizes the geometrical parameters of the tested components and provides the reference names used in the paper. All the waveguides are described with the reference Gxx where $\mathrm{xx}$ is a specific number. The letter " $T$ " is added if the waveguide supports a taper.

\section{Laboratory validation}

The laboratory workbench aims at testing and analyzing the single-mode behavior and the propagation features of the manufactured waveguides. The optical layout of the injection 

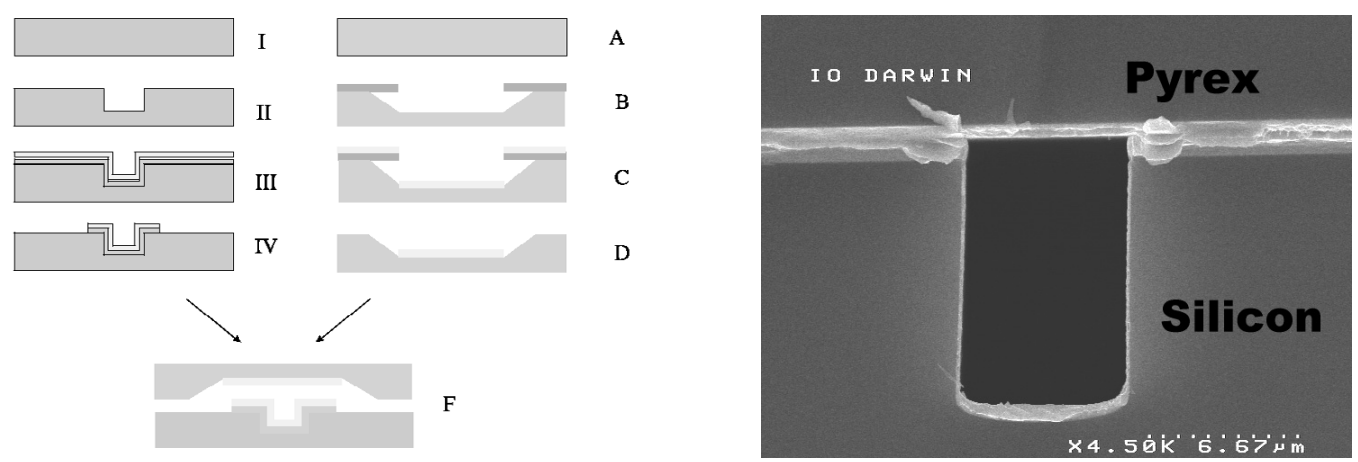

Fig. 1. Left panel: process flow of HMW manufacturing. I: Silicon 4-inch wafer - II: Guide definition by photo-lithography and RIE III: Thermal oxidation and gold deposition - IV: Gold and silica wet etching - A: Pyrex ${ }^{T M}$ 4-inch wafer - B: Photo-lithography and Pyrex ${ }^{T M}$ wet etching - C: Gold deposition - D: Photo-resist stripping - F: Anodic bonding. Right panel: photograph of a HMW input using a scanning electron microscope. The Pyrex cover is maintained by anodic bonding on the silicon substrate. The etching depth is $10 \mu \mathrm{m}$. The gold deposition is thicker on the bottom of the waveguide than the lateral walls.

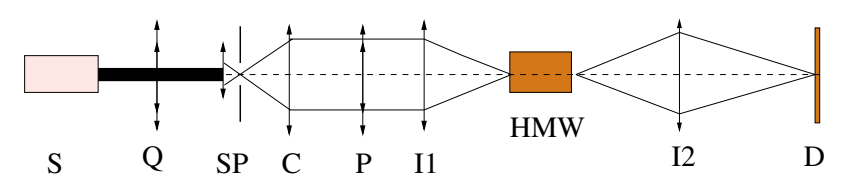

Fig. 2. Layout of the injection workbench at $\lambda=10.6 \mu \mathrm{m}$. S: $\mathrm{CO}_{2}$ laser source - Q: Quarter wavelength ZnSe plate - SP: $50 \mu \mathrm{m}$ pinhole spatial filter $-\mathrm{P}$ : Grid polarizer $-I_{1}$ : Injection lens - HMW: Hollow Metallic Waveguide - $I_{2}$ Imaging lens - D: Detector array. The polarizer $\mathrm{P}$ can be placed after the component HMW.

workbench is provided in Fig. 2. The chip containing the channel waveguides is supported on a three-axis positioner with fine step resolution $(\sim 1 \mu \mathrm{m})$ to co-align the components with the optical axis. Considering the dimensions of the waveguide aperture, a major constraint is put on the optical quality of the input beam. Therefore, we use aspherical fast optics with numerical aperture $f / 1.15$ to focus the beam on the waveguide aperture, providing a spot with $30 \mu \mathrm{m}$ full-width. A beamexpander permits filling of the 22-mm clear aperture of the focalization lens. The output flux is collected with a $f / 2.29$ lens that images the waveguide output on the camera focal plane. The detection stage supports an uncooled $320 \times 240$ microbolometer array detector sensitive from $8 \mu \mathrm{m}$ to $12 \mu \mathrm{m}$. A grid polarizer is placed either before the component (i.e. into the collimated beam) or after the component to select the desired polarization to be analyzed. The extinction ratio of the polarizer at $\lambda=10 \mu \mathrm{m}$ is $185: 1$, which is equivalent to a polarization degree of $99.4 \%$. The Gaussian beam distribution of the laser is highly flattened since the collimator $\mathrm{C}$ with $f=254 \mathrm{~mm}$ is overfilled. Thus, the spot at the waveguide input can be considered as diffraction-limited.

The linear polarization of the $\mathrm{CO}_{2}$ laser has been converted into elliptical polarization by adding a quarter-wave plate in the ray path. This forces any polarization direction at the waveguide input. The elliptical polarization of the source is characterized by the curve in the left panel of Fig. 5 that plots the calibrated flux level of the source as a function of the angular position of the polarizer.

The $300 \mu \mathrm{m}$ spacing between the different channel waveguides is used as a qualitative validation criterion for the observation of the waveguide output. Indeed, we can observe successive bright spots when translating the chip by the separation of $300 \mu \mathrm{m}$, which confirms that the detected flux has been guided along the waveguide. For quantitative relative flux measurements, a flat field correction is applied and the mean level of the thermal background is subtracted as discussed below.

For each image with a flux $I_{\text {raw }}$ per pixel, a dark image $I_{\text {dark }}$ corresponding to the response of the array to a spatially uniform source is recorded. The flat field correction is applied for each single pixel by computing a corrected image $I_{\text {flat }}$ given by

$I_{\text {flat }}=\frac{I_{\text {raw }}}{I_{\text {dark }} / \tilde{I}_{\text {dark }}}$

where $\tilde{I}_{\text {dark }}$ is the pixel average value of the dark image. The mean level of the thermal background is obtained by computing the pixel average $\tilde{m}_{\mathrm{BG}}$ of a sub-frame of $I_{\text {flat }}$ where the background is uniform and where no signal is detected. The final corrected image $I_{\text {cor }}$ is given by

$I_{\text {cor }}=I_{\text {flat }}-\tilde{m}_{\mathrm{BG}}$.

As an example, Fig. 3 presents a cross-sectional view of the correction stage as well as a calibrated image of a waveguide output. The artifacts visible in the left panel of Fig. 3 are filtered out with the applied correction.

To perform transmission measurements of the waveguide, we compare the calibrated flux without the waveguide $I_{\text {cor }}^{\text {out }}$ and the calibrated flux through the waveguide $I_{\text {cor }}^{\text {in }}$. The estimated relative transmission of the waveguide is thus

$T_{\text {est }}=\frac{I_{\text {cor }}^{\text {in }}}{I_{\text {cor }}^{\text {out }}}$

where $I_{\text {cor }}^{\text {in }}$ is the transmitted power through the waveguide and $I_{\text {cor }}^{\text {out }}$ is the transmitted power without the waveguide. $T$ is defined as the total throughput of the component.

The time variations of the source and of the the thermal background in the laboratory have been measured over a timescale of $1 \mathrm{~h}$ to evaluate the achievable signal-to-noise ratio. The characterization results in a variation of $0.2 \%$ for the thermal background and $1.5 \%$ for the laser signal. 

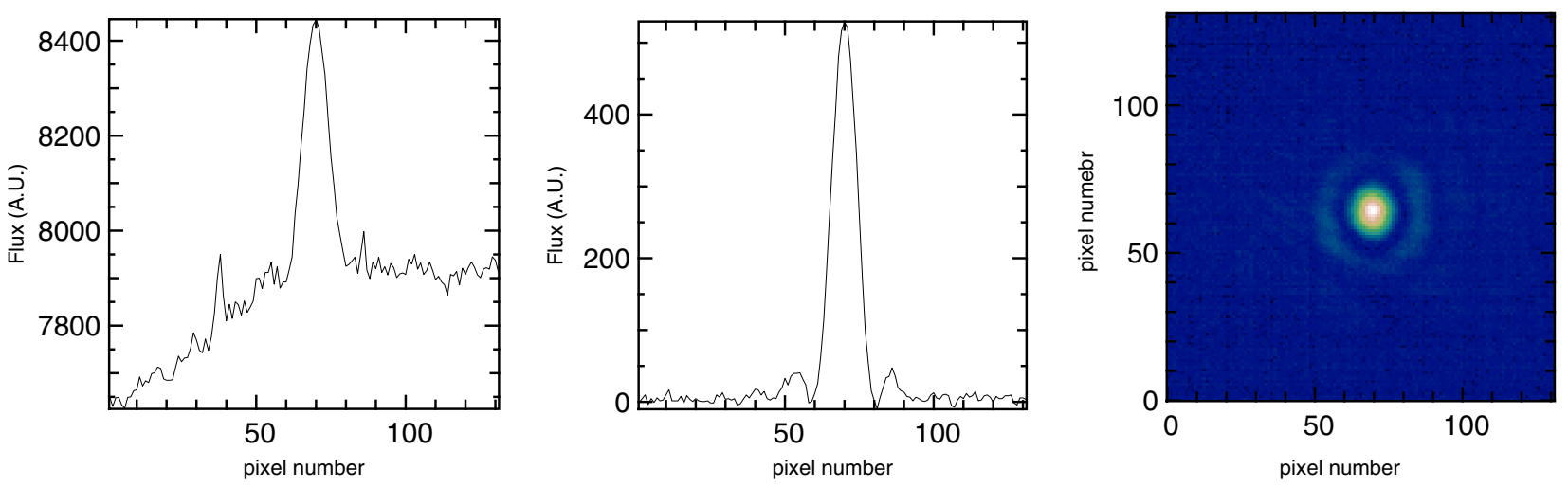

Fig. 3. Signal correction with flat and background suppression. The left panel of the figure shows the raw signal from the camera, which contains artifacts due to the response of each pixel. In the center panel, the correction described in Sect. 4 has been applied, which results in a uniform distribution around the central peak. Once the background mean level has been subtracted, the signal is affected by its zero-mean Poisson noise. The right panel of the figure presents the diffraction-limited spot corresponding to the output flux of the waveguide.

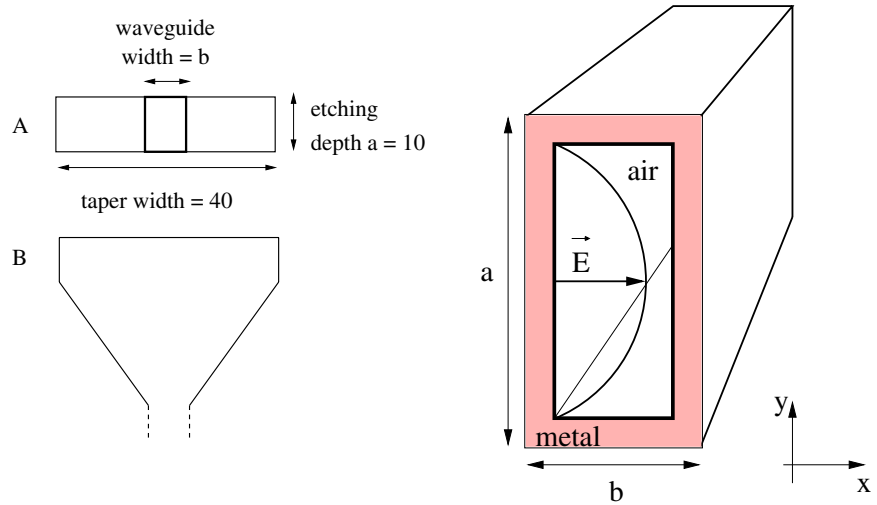

Fig. 4. Left panel: front view (A) and upper view (B) of a taper. The etching depth is fixed at $10 \mu \mathrm{m}$ and the taper width is $40 \mu \mathrm{m}$. The waveguide width varies according to Table 1. Right panel: geometry of Hollow Metallic Waveguides designed to be single-mode. The dominant mode is the fundamental $\mathrm{TE}_{10}$ whose distribution follows a cosine $y$-dependent distribution law.

\section{Experimental results}

\subsection{Characterization of the modal behavior by polarization tests at $\lambda=10.6 \mu \mathrm{m}$}

As presented in Sect. 3, a rectangular HMW designed to be single-mode supports only one state of polarization while a multi-mode HMW presents at least two orthogonal states of polarization with different field distributions. According to its geometry, the fundamental mode is the $\mathrm{TE}_{10}$ with the electric field oriented along the $x$-axis (see Fig. 4) and the potential first higher order mode is the $\mathrm{TE}_{01}$ with the electric field oriented along the $y$-axis (Jordan \& Balmain 1985). To test the modal behavior of the waveguides, we have placed the grid polarizer after the component output to analyze the polarization state. Depending if a waveguide is single-mode or multi-mode, flux will be detected for any angular position (multi-mode) or only for a specific angular position (single-mode) of the polarizer. Analyzing the output polarization state is a simple method that gives unbiased information to discriminate between $\mathrm{TE}_{10}$ and $\mathrm{TE}_{01}$ propagating modes.
The theoretical expected intensity distribution at a singlemode waveguide output is (Jordan \& Balmain 1985)

$I_{\text {out }}(\theta) \propto I_{0} \sin ^{2}(\theta)$

where $I_{0}$ is a constant intensity and $\theta$ the angle between the $y$-axis and the tested polarization direction. The plot in the center panel of Fig. 5 shows the calibrated flux in arbitrary units measured after the waveguide as a function of the angular position of the grid polarizer. This result is obtained with the sample G36-T (see Table 1) designed to be single-mode at $\lambda=10.6 \mu \mathrm{m}$.

The angular positions $0^{\circ}$ and $180^{\circ}$ correspond to the polarizer oriented along the $y$-axis in Fig. 4, which theoretically matches the total extinction of the electric field oriented orthogonally. The plot shows that the electric field can be totally extinguished experimentally at these two extreme angular positions. The first higher order mode being $\mathrm{TE}_{01}$ according to the geometry of G36-T, this result shows that no electrical field oriented along the $y$-axis can be excited at a wavelength of $10.6 \mu \mathrm{m}$, which confirms the single-mode behavior of the waveguide.

Polarization tests also have been made for waveguides designed to be multi-mode at $\lambda=10.6 \mu \mathrm{m}$. The results on the tested sample G42-T (see Table 1) have been reported here. The propagation modes theoretically existing in the cavity are the fundamental mode $\mathrm{TE}_{10}$ with its electric field oriented along the $x$-axis, the mode $\mathrm{TE}_{01}$ with the electric field oriented along the $y$-axis, and the modes $\mathrm{TE}_{11}$ and $\mathrm{TM}_{11}$ with a distribution of the electric field both in the $x$ and $y$ directions (Jordan \& Balmain 1985). Since $\mathrm{TE}_{11}$ and $\mathrm{TM}_{11}$ are odd modes, it is assumed that no energy will be coupled to those modes if the excitation field is centered on the waveguide aperture. The right panel of Fig. 5 shows the flux measured after the sample G42-T as a function of the angular position of the polarizer.

The curve presents a strong decrease around $100^{\circ}$, but for any position of the polarizer between $0^{\circ}$ and $180^{\circ}$ it is always possible to detect flux transmitted through the waveguide. The minimum value is about 150 , which is above the noise limit. Thus, no single-polarization state can be measured with G42-T, which shows that higher order modes can propagate into the waveguide. 

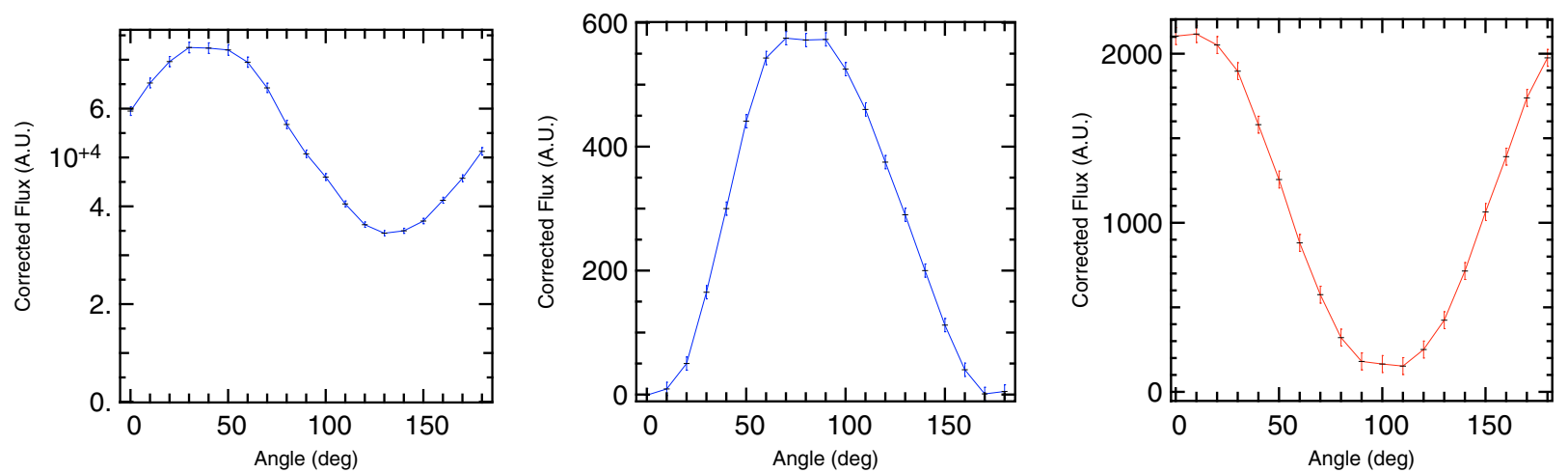

Fig. 5. a) Characterization curve of the elliptically polarized source. b) Polarization measurements for the single-mode waveguide G36-T at $\lambda=10.6 \mu \mathrm{m}$ with $a=10 \mu \mathrm{m}$ and $b=5.1 \mu \mathrm{m}$. The output flux can be totally extinguished at angular positions $0^{\circ}$ and $180^{\circ}$ with $\sim 2.5 \%$ accuracy c) Polarization measurements for the multi-mode waveguide G42-T at $\lambda=10.6 \mu \mathrm{m}$ with $a=10 \mu \mathrm{m}$ and $b=9.2 \mu \mathrm{m}$. A strong variation of the flux level is observed but it is not possible to achieve a total extinction of the output flux for any of the polarization direction. For the three graphs, the flux level is reported in arbitrary units (AU).

For the single-mode G36-T component, the error bars measured for the total extinction are limited by the thermal background fluctuations ( $\sim 16 \mathrm{AU}$ in Fig. 5). The maximum flux level is about $600 \mathrm{AU}$, which guarantees an accuracy of $\sim 2.5 \%$ on the measurement. The maximum flux detected with the multi-mode waveguide G42-T ( 2100 AU) is three to four times the maximum flux detected with the single-mode waveguide G36-T ( $600 \mathrm{AU})$. This can be explained by power coupling on higher order modes that can propagate into the multimode waveguide, while they are filtered by the single-mode waveguide.

In Table 1 we give the results of the modal characterization of a precise set of waveguides at $\lambda=10.6 \mu \mathrm{m}$ based on polarization tests. Our results show that a cut-off is experimentally observed for waveguide widths above $w=5.1 \mu \mathrm{m}$. This confirms the single-mode range predicted theoretically in Sect. 3.

The analysis based on polarization tests offers a quantitative measurement of the modal behavior that is based on flux level measurements. This method can be compared to the nearfield imaging method used in Laurent (2003) to characterize fibers for the near-infrared, which is based on a qualitative analysis of the output intensity distribution. With this last method, the discrimination between the fundamental and the first mode is more difficult when analyzing the field spatial distribution rather than implementing a flux measurement.

\subsection{Waveguide transmission}

In a simple approach, the total throughput $T$ of a channel hollow metallic waveguide can be separated into 1) input coupling efficiency $C_{\mathrm{in}, \mathrm{i}}$ in the mode $\mathrm{i}$; 2) propagation losses $P_{\mathrm{i}}$ of the mode i; 3 ) output coupling efficiency $C_{\text {out,i }}$ to the mode i; 4) a quality factor $\eta_{\mathrm{i}}$ through the expression

$T=\sum_{\mathrm{i}=1}^{\mathrm{n}} C_{\mathrm{in}, \mathrm{i}} \cdot P_{\mathrm{i}} \cdot C_{\mathrm{out}, \mathrm{i}} \cdot \eta_{\mathrm{i}}$

which simply becomes in the single-mode case

$T(\%)=C_{\text {in }} \cdot P \cdot C_{\text {out }} \cdot \eta$.
The term $P$ is dependent on the waveguide length. The quality factor $\eta$ includes a term of impedance mismatch comparable to Fresnel losses for near-infrared fibers and the losses due to technological imperfections at the taper input and output that degrade the coupling efficiency and that are not considered when computing the overlap integral. $\eta$ is dependent on the manufacturing process and can vary from one waveguide to another. The term $P \cdot \eta$ is defined as the excess losses and corresponds to the physical quantity that can be measured experimentally. It is therefore a worst-case value of the propagation losses $P$ of the waveguide.

The input and output coupling efficiencies must then be evaluated to estimate the propagation term $P \cdot \eta$. They have been estimated numerically by computing the two-dimensional overlap integral between the excitation field and the dominant mode profiles at the waveguide input and output. The excitation field at the waveguide input is a plane wave focused by a lens with focal length $f$ and a clear aperture $D$. The shape of the field can be modelized with an Airy function given by

$E_{\mathrm{exc}}\left(x, y, \frac{f}{D}\right)=2 \frac{J_{1}\left(\frac{D}{f} \cdot \frac{\pi \sqrt{(x-d)^{2}+(y-e)^{2}}}{\lambda}\right)}{\left(\frac{D}{f} \cdot \frac{\pi \sqrt{(x-d)^{2}+(y-e)^{2}}}{\lambda}\right)}$

where $E_{\text {exc }}$ stands for the electric excitation field amplitude, $f$ is the focal length of optics $I_{1}$ (see Fig. 2) and $D$ its clear aperture. $J_{l}$ is the first order Bessel function, $d$ and $e$ are the lateral displacement with respect to the center of the waveguide aperture respectively in the $x$ and $y$ directions. Since the $E_{\text {exc }}$ has a radial symmetry and the waveguide aperture has two symmetry axes, it can be shown that the maximum coupling efficiency occurs when the excitation field is centered on the waveguide input (i.e. $d=e=0$ ). Losses $\leq 1 \%$ occur for a displacement $d$ or $e$ of $1-\mu \mathrm{m}$ (Schanen 2003). Thus, in the next sections the coupling efficiencies are considered to be computed for a centered excitation field.

Due to the quality factor $\eta$, the experimental coupling efficiency might differ from the theoretical values. It is possible to evaluate the order of variation between observation and theory 


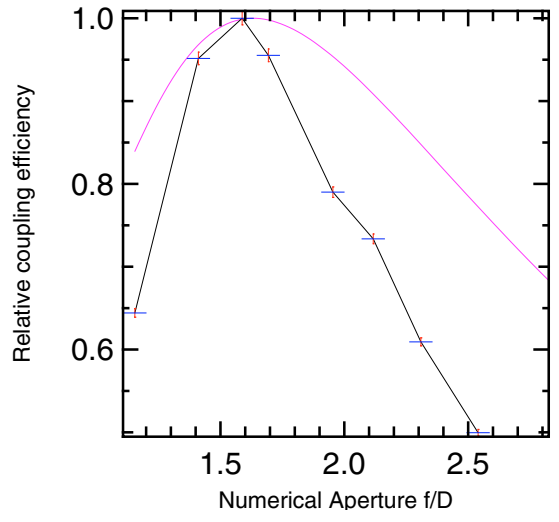

Fig. 6. Relative coupling efficiency as a function of the incident beam aperture. The crosses give the experimental results and the other curve is obtained with the model.

by changing the diameter (i.e. the numerical aperture) of the injection spot given in Eq. (7) and measuring the variations of the transmitted flux.

Figure 6 compares the normalized coupling efficiency at an aperture with a taper obtained with the model approach and with the experimental data. The coupling efficiency curve has been normalized to one because it is only possible to make a relative comparison of the different data via differential flux measurements. It is not possible to extract the absolute values of the efficiencies with our current test configuration. Doing this test with a tapered waveguide has the advantage of experimentally observing a maximum for the coupling efficiency curve, which would not have been possible testing a waveguide without a taper for which maximum occurs below $f / 0.5$. The crosses show that a maximum coupling efficiency is obtained experimentally for $f / D \approx 1.6$, which matches the value computed with the model. This shows that the method of the overlap integral can be applied in our case. Aside from the maximum coupling, the experimental curve presents a significant degradation of up to $20 \%$ relative to the theory. This shows the importance of the quality factor $\eta$ in degradation of the coupling efficiencies.

We report in Table 1 the results of the characterization phase of HMW at $\lambda=10.6 \mu \mathrm{m}$. In Eq. (6), $T$ is measured experimentally, $C_{\mathrm{in}}$ and $C_{\text {out }}$ are obtained numerically which permits us to extract the excess losses $P \cdot \eta$. The excess loss is the only transmission feature that can be extracted from experimental measurements and is therefore given in $\mathrm{dB}$ while the propagation losses are given in $\mathrm{dB} \mathrm{mm}^{-1}$.

The output coupling efficiency has been computed with the following parameters: the imaging optical system has a focal length $f=38.1 \mathrm{~mm}$ and a clear diameter $D=16.5 \mathrm{~mm}$, which gives a numerical aperture $f / 2.29$. At $\lambda=10.6 \mu \mathrm{m}$, the imaging optics are limited to $28 \mu \mathrm{m}$ resolution and a typical aperture of $10 \mu \mathrm{m} \times 10 \mu \mathrm{m}$ will not be resolved. This results in the diffraction-limited energy distribution shown in Fig. 3. As a consequence, a Bessel distribution is assumed with the numerical aperture of $f / 2.29$ to compute the output coupling efficiency $C_{\text {out }}$.

The injection tests have been carried out with the workbench configuration presented in Sect. 4 in which the injection lens $I_{1}$ has a numerical aperture of $f / 1.15$. Although this aperture is not the optimal one when considering $40 \mu \mathrm{m} \times 10 \mu \mathrm{m}$ tapers, it is the best option for waveguides without tapers.

For multi-mode waveguides, it is not possible to separate experimentally the excess losses of each mode with our setup. However it is still possible to compare the total throughput as is done in Table 1. When considering tapered waveguides, a clear transition can be observed in transmission as predicted by the theory. Filtering the higher order modes results in a significant drop in flux, which corresponds to the transition between the single-mode and multi-mode regime. The same aspect is encountered with traditional dielectric infrared fibers.

Theoretically, propagation losses for rectangular singlemode waveguides with $a=10 \mu \mathrm{m}$ are $\sim 0.8 \mathrm{~dB} \mathrm{~mm}^{-1}$ at $10.6 \mu \mathrm{m}$ (see Schanen 2003; and Appendix A). A static conductivity of $\sigma_{0}=4.1 \times 10^{7} \mathrm{~S} \mathrm{~m}^{-1}$ has been assumed for the gold coating. Experimentally, excess losses of $2.5 \mathrm{~dB}$ to $4 \mathrm{~dB}$ over 1-mm are measured in this study. This difference results from a poor quality factor at the waveguide input and output that strongly affects the coupling efficiency. The important dispersion in losses for the sample G46 in Table 1 could be explained with a similar dispersion in the technological repeatability, which could be improved in a subsequent technological run. However, the clear distinction in transmission between single-mode and multi-mode waveguides shows that the quality factor does not prevent us from assessing the modal properties of those waveguides. New considerations on waveguide losses are presented in Sect. 6.

\section{Discussion}

The analysis through polarization tests showed the single-mode behavior of waveguides at $10.6 \mu \mathrm{m}$, estimating it with $\sim 2.5 \%$ accuracy due to background fluctuations. The first measurement confirms that conductive waveguides are suitable for single-mode waveguides in the mid-infrared.

The optical quality of the waveguide input and output facets (included within the factor $\eta$ in the paper) greatly affects the excess losses. At the moment, the chips containing the waveguides are cut out from the wafer using dicing and cleaving processes, which result in unexpected irregularities around the waveguide inputs. Different technological options have been considered, which should reduce the dispersion on quality and then improve the technological repeatability.

The experimental results have shown the fundamental importance of coupling optimization at the waveguide input and output. In this first technological run, a strong priority has been set on manufacturing rather than on design, so the computed coupling efficiencies in Table 1 are not optimized. We are confident that coupling efficiencies can be improved through a more stringent design of the tapers in the next run.

One of the most recurring issues for spatial or modal filtering is the effects of chromatism. With optical and infrared waveguides, modal filtering can be implemented in a spectral range where the device is single-mode. We have seen that for conductive rectangular waveguides with dimensions $a$ and $b=a / 2$, the single-mode domain is given for $a \leq \lambda \leq 2 a$, which represents a $66 \%$ bandwidth centered on $\lambda=1.5 a \mu \mathrm{m}$. 
Thus, this property is used to divide the [4-20 $\mu \mathrm{m}]$ band into a relatively low number (three or four) of single-mode sub-bands.

However, it is not recommended to apply this reasoning straightforwardly since the propagation losses within the same sub-band are highly chromatic. Schanen (2003) has extended the well-established loss calculations in the millimeter regime to the mid-infrared domain using Drude's model approach for the metallic coating. Appendix A presents the main steps of the calculation for this approach. The attenuation coefficiant of the dominant mode $\mathrm{TE}_{10}$ is expressed as a function of $\lambda, \lambda_{\mathrm{c}}$, and $(n$, $k$ ) the real and imaginary indeces of gold in the mid-infrared. The conclusion are: first, the average losses in a given singlemode sub-band are higher at shorter wavelength than at longer ones; secondly, within the same sub-band, losses are minimized for wavelengths close to the lower cut-off (i.e. $\lambda_{\mathrm{c}}=a$ ) and increase continuously when $\lambda$ approaches the higher cut-off (i.e. $\left.\lambda_{\mathrm{c}}=2 a\right)^{1}$. Dividing the range $[4-20 \mu \mathrm{m}]$ is therefore a trade-off between the bandwidth and the average losses of a single-mode sub-band.

Following this analysis, a trade-off is proposed dividing the $[4-20 \mu \mathrm{m}]$ band into four single-mode sub-bands $B_{1}=$ [4-6 $\mu \mathrm{m}], B_{2}=[6-10 \mu \mathrm{m}], B_{3}=[10-16 \mu \mathrm{m}]$ and $B_{4}=$ [16-20 $\mu \mathrm{m}]$ using as modal filters HMW with $a$ verifying $a=4 \mu \mathrm{m}$ for $B_{1}, a=6 \mu \mathrm{m}$ for $B_{2}, a=10 \mu \mathrm{m}$ for $B_{3}$ and $a=16 \mu \mathrm{m}$ for $B_{4}{ }^{2}$. The computed average losses for each sub-band are respectively $3.5 \mathrm{~dB} \mathrm{~mm}^{-1}$ for $B_{1}, 2.7 \mathrm{~dB} \mathrm{~mm}^{-1}$ for $B_{2}, 1.5 \mathrm{~dB} \mathrm{~mm}^{-1}$ for $B_{3}$ and $0.6 \mathrm{~dB} \mathrm{~mm}^{-1}$ for $B_{4}$.

Compared to the computed performances, the transmission values presented in Table 1 are also affected by the surface roughness of the metallic walls, which is not taken into account yet in the loss model. To date a gold coating with roughness lower than $50 \mathrm{~nm}$ rms can be deposited but the roughness increases during the anodic bonding process. A better control of this last step is achievable, decreasing the roughness.

Schanen (2003) has shown that an efficient filtering of $10^{-7}(\sim 70 \mathrm{~dB})$ is reached after a $10 \lambda$ length $(\sim 100 \mu \mathrm{m})$ of the waveguide (see Appendix B). This implies that integrated optics functions (e.g. an IO beam combiner) could be implemented over short propagation distances, typically less than 1-mm length, reducing the propagation losses. Making shorter functions has already been addressed and is technically achievable.

HMW present interesting polarization properties with respect to polarization control presented in Sect. 1. A single-mode rectangular metallic waveguide presents a singlepolarization behavior, which means the implemented device is able to force a linear polarization at its input. Under these conditions, the beams combined in the waveguide do not present polarization mismatching. Since the differential rotation of polarization planes has to be controlled down to $0.002 \mathrm{rad}$ to achieve a $10^{6}$ rejection ratio (Mennesson et al. 2002), singlepolarization waveguides remove this constraint, although at the expense of power transmission.

\footnotetext{
${ }^{1}$ The attenuation coefficient of a propagating mode theoretically tends to infinity when $\lambda$ equals the cut-off wavelength.

2 The fourth waveguide is single-mode up to $\lambda=32 \mu \mathrm{m}$, but the definition of the Darwin band limits it to $\lambda=20 \mu \mathrm{m}$.
}

\section{Conclusions}

This study has focused on the feasibility of manufacturing rectangular Hollow Metallic Waveguides to be used as modal filters because of their single-mode behavior. This offers an original instrumental solution in a spectral range where similar possibilities are limited. The demonstrated single-mode behavior is considered as major result of this study.

A quantitative measurement based on experimental polarization analysis has shown the single-mode behavior of the manufactured waveguides with $\sim 2.5 \%$ accuracy and has been compared with results obtained on multi-mode waveguides. Single-mode HMW show that a single-polarization state can be maintained in the waveguide, which is an advantage for polarization control in nulling interferometry. Improving the total throughput of such waveguides is a matter of increasing the coupling efficiencies at waveguide inputs and outputs. This can be increased significantly through the design of well-adapted horns.

Single-mode channel waveguides could be extended to the manufacturing of planar integrated components. Those devices could ensure simple interferometric functions (recombination, photometric control) whose compactness and stability would be very useful in future interferometry space missions.

\section{Appendix A: Derivation of the theoretical propagation losses of the $\mathrm{TE}_{10}$ mode}

This annex presents the main results contained in Schanen (2003). In the microwave regime - i.e. for frequencies ranging from 1 to $1000 \mathrm{GHz}$ - the computation of the theoretical propagation losses is based on the model of the "skin effect" (Rizzi 1988). It is well known that the tangential electric field at the surface of a perfect metallic medium is zero. For a real metallic coating, the finite conductivity induces the presence of a small tangential component of the electric field in the metal. As a consequence, some power flows through the lateral walls of the waveguide.

To extract the attenuation factor of the guided mode, we identify the average guided power lost between the coordinates $z=0$ and $z=L$ of the propagation axis with the power flowing through the metallic walls of an equivalent length. In this approach, the static conductivity of the metal $\sigma_{0}$ is considered as long as we remain in the microwave regime since the frequency of the electromagnetic wave is much lower than the collision frequency of the electrons $\left(\sim 10^{13} \mathrm{~Hz}\right.$, Quéré 1988). In the mid-infrared domain domain, the radiation frequency is much higher $\left(\sim 3 \times 10^{13} \mathrm{~Hz}\right)$ and the conductivity of the metal $\sigma$ becomes frequency-dependent following the Drude model (Quéré 1988). This has been taken into account in the proposed approach.

The power dissipated in the walls $W_{\mathrm{J}}$ between $z=0$ and $z=L$ is obtained by computing the flux of the Poynting vector across the waveguide section at the corresponding $z$-coordinates. This results in

$W_{\mathrm{J}}=\left(1-\exp \left(-2 \alpha_{\mathrm{g}} L\right)\right) \int_{\text {section }}\left(\boldsymbol{E} \times \boldsymbol{H}^{*}\right) \cdot z \mathrm{~d} x \mathrm{~d} y$ 
where $\boldsymbol{E}$ and $\boldsymbol{H}$ are respectively the electric and magnetic field, $\alpha_{\mathrm{g}}$ the attenuation factor. Using the equations of $\boldsymbol{E}$ and $\boldsymbol{H}$ fields for the $\mathrm{TE}_{10}$ fundamental mode (Jordan \& Balmain 1985), Eq. (A.1) becomes

$W_{\mathrm{J}}=\left(1-\exp \left(-2 \alpha_{\mathrm{g}} L\right)\right) C^{2} \frac{2}{\lambda^{2}} b a^{3} \sqrt{\frac{\mu_{0}}{\epsilon_{0}}} \sqrt{1-\frac{\lambda^{2}}{\lambda_{c}^{2}}}$

where $C$ is a field constant, $\lambda$ the operating wavelength, $\lambda_{\mathrm{c}}$ the $\mathrm{TE}_{10}$ cut-off wavelength, $a$ and $b$ the waveguide dimensions.

To compute the tangential component of the $\boldsymbol{E}$ field in the metal, we employ the Maxwell equation

$\frac{\mathrm{d}}{\mathrm{d} z} H_{x}-\frac{\mathrm{d}}{\mathrm{d} x} H_{z}=-\jmath \omega \epsilon E_{y}+\sigma E_{y}$

where $\epsilon$ and $\sigma$ are the complex permittivity and conductivity of the metal obtained through the Drude model of a metal, omega the radiation frequency. At infrared wavelengthes, the commonly used approximation $\operatorname{Re}[\sigma] \gg \operatorname{Re}[\epsilon] \omega$ is no longer valid and both terms must be maintained in Eq. (A.3). The wave vector $\boldsymbol{k}$ of $\mathrm{TE}_{10}$ mode presents a component $k_{z}$ along the propagation axis, as expected in the case of a perfect metal, and a component along the $y$-axis (see Fig. 4) corresponding to a slight penetration of the $\boldsymbol{E}$ field in the metal. Quantities $k_{z}$ and $k_{x}$ are determined using the formalism of the reflection of electromagnetic radiation at a metallic surface. We derive

$k_{z}=j \frac{\omega}{\mathrm{c}} \sqrt{1-\left(\frac{\lambda}{\lambda_{\mathrm{c}}}\right)^{2}}$

$k_{x}=J \frac{\omega}{\mathrm{c}} \sqrt{(n-\jmath \kappa)^{2}-\left(1-\left(\frac{\lambda}{\lambda_{\mathrm{c}}}\right)^{2}\right)}$

$N=n-j \kappa$ is the complex refractive index of the metal. Quantities $n$ and $k$ at $\lambda=10 \mu \mathrm{m}$ are from Ordal et al. (1983). Solving Eq. (A.3), we obtain an expression of the $\boldsymbol{E}$ field in the metal following

$E_{x}=C \frac{1}{\jmath \omega \epsilon-\sigma} \frac{k_{y}^{2}+k_{z}^{2}}{k_{y}} \exp (-\jmath \omega t) \exp \left(k_{y} y+k_{z} z\right)$.

The expressions of the conductivity $\sigma$ and permittivity $\epsilon$ follow

$\sigma=\frac{\sigma_{0}}{(1-\jmath \omega \tau)}$

$\sigma_{0}=\epsilon_{0} \epsilon_{\mathrm{r}} \tau\left(\omega_{\mathrm{p}}\right)^{2}$

where $\omega_{\mathrm{p}}$ is called the "plasma frequency" and caracterizes the media (Ordal et al. 1983). The power flowing through the metallic walls is obtain in analogy to Eq. (A.1) through

$P_{\mathrm{J}}=\frac{1}{2} \operatorname{Re}[\sigma] \int_{V} \boldsymbol{E}_{\boldsymbol{x}} \cdot \boldsymbol{E}_{\boldsymbol{x}}{ }^{*} \mathrm{~d} V$

where $\operatorname{Re}[$ ] is the "real part" operator. The quantity $d V$ is the elementary volume that takes into account the walls surface and the penetration depth of the $\boldsymbol{E}$ field in the metal. We derive the following expression for $P_{\mathrm{J}}$

$$
\begin{aligned}
P_{\mathrm{J}}= & \frac{1}{2} C^{2} \frac{\operatorname{Re}[\sigma]}{\sigma^{2}+(\omega \epsilon)^{2}}\left|\frac{\left(k_{x}\right)^{2}+\left(k_{z}\right)^{2}}{k_{x}}\right|^{2} \\
& \times b \frac{1}{2 \operatorname{Re}\left[k_{x}\right]} \frac{1-\exp \left(-2 \alpha_{\mathrm{g}} L\right)}{2 \alpha_{\mathrm{g}}} .
\end{aligned}
$$

From Eqs. (A.7) and (A.8), the term $\left(\operatorname{Re}[\sigma] /\left(|\jmath \omega \epsilon-\sigma|^{2}\right)\right)$ can be simplified to $1 / \sigma_{0}$, where $\sigma_{0}$ is the static conductivity of the metal. Using the fact that

$\kappa^{2}-n^{2}>1>1-\left(\frac{\lambda}{\lambda_{c}}\right)^{2}$

we derive the following expressions

$\operatorname{Re}\left[k_{x}\right]=\frac{\omega_{\mathrm{c}}}{c} \kappa\left(1+\frac{\left(1-\left(\frac{\lambda}{\lambda_{\mathrm{c}}}\right)^{2}\right)}{2\left(n^{2}+\kappa^{2}\right)}\right)$

and

$$
\begin{aligned}
& \left|\frac{\left(k_{x}\right)^{2}+\left(k_{z}\right)^{2}}{k_{x}}\right|^{2}= \\
& \frac{\omega_{\mathrm{c}}}{c}\left(n^{2}+\kappa^{2}+2\left(1-\left(\frac{\lambda}{\lambda_{\mathrm{c}}}\right)^{2}\right) \frac{\left(n^{2}-\kappa^{2}\right)}{\left(n^{2}+\kappa^{2}\right)}\right) .
\end{aligned}
$$

Equations (A.12) and (A.13) are used to derive the complete analytical expression of Eq. (A.10). The attenuation factor $\alpha_{\mathrm{g}}$ is now obtained by assuming that $W_{\mathrm{J}}=2 P_{\mathrm{J}}$. The attenuation factor, given in $\mathrm{Np} \mathrm{m}^{-1}$ (Nepers per meter) gives

$$
\begin{aligned}
\alpha_{\mathrm{g}}= & \frac{\pi}{2} \frac{1}{\sigma_{0}} \sqrt{\frac{\epsilon_{0}}{\mu_{0}}}\left(\frac{n^{2}+k^{2}+2\left(1-\left(\frac{\lambda}{\lambda_{\mathrm{c}}}\right)^{2}\right) \frac{n^{2}-k^{2}}{n^{2}+k^{2}}}{k\left(1+\frac{1-\left(\frac{\lambda}{\lambda_{\mathrm{c}}}\right)^{2}}{2\left(n^{2}+k^{2}\right)}\right)}\right) \\
& \times \frac{\lambda}{a^{3}} \frac{1}{\sqrt{1-\left(\frac{\lambda}{\lambda_{\mathrm{c}}}\right)^{2}}} \cdot
\end{aligned}
$$

Equation (A.15) is converted into more useful units $\mathrm{dB} \mathrm{m}^{-1}$ by multiplying the expression by factor 8.68 (Jordan \& Balmain 1985).

\section{Appendix B: Derivation of filtering length for higher order mode suppression}

We consider a rectangular HMW with $a=2 b$. The single mode domain ranges from $\lambda=a$ to $\lambda=2 a$. The cut-off wavelenght of the first higher order mode $\mathrm{TE}_{01}$ is $\lambda_{\mathrm{c}}=a=2 b$.

In the single-mode regime, the propagation constant $\gamma$ of the $\mathrm{TE}_{01}$ mode as it appears in $\exp (-\gamma z)$ is real and equals

$\gamma=\frac{2 \pi}{\lambda} \sqrt{\left(\frac{\lambda}{\lambda_{\mathrm{c}}}\right)^{2}-1}$

with $\lambda>\lambda_{\mathrm{c}}$. The term $\gamma$ is transformed into the attenuation factor $\alpha$ of the mode in $\mathrm{dB} \mathrm{m}^{-1}$ through (Jordan \& Balmain 1985)

$\alpha=8.68 \times \gamma$.

Assuming a HMW with dimensions $a=2 b=10 \mu \mathrm{m}$, the attenuation $\alpha$ factor is lower as $\lambda$ is closer to $\lambda_{\mathrm{c}}$. Let us consider the worst case with $\lambda=10.1 \mu \mathrm{m}$. At this wavelength, Eq. (B.1) provides an attenuation factor $\alpha=0.76 \mathrm{~dB} \mu \mathrm{m}^{-1}$. Thus the waveguide length required to attenuate the higher order mode by $70 \mathrm{~dB}\left(\sim 10^{-7}\right)$ is $92 \mu \mathrm{m}$. As the wavelength increases, the attenuation factor increases as well and the required filtering length becomes shorter. 
Acknowledgements. This work was supported by a European Space Agency (ESA) under contract 16847/02/NL/S Fe and supported by funding from the French Space National Agency (CNES) and Alcatel Space.

\section{References}

Angel, J. R. P., Cheng, A. Y. S., \& Woolf, N. J. 1986, Nature, 322, 341 Beichman, C. 2001, Bull. Am. Astron. Soc., 33, 860

Bordé, P. J., Perrin, G. S., Nguyen, C. T., et al. 2003, in Interferometry for Optical Astronomy II, ed. W. A. Traub, Proc. SPIE, 4838, 273

Bracewell, R. N. 1978, Nature, 274, 780

Bracewell, R. N., \& McPhie, R. H. 1979, Icarus, 38, 136

Coudé du Foresto, V., \& Ridgway, S. T. 1992, in High-Resolution Imaging by Interferometry, 731

Fridlund, M. 2000, in Bioastronomy 99, ASP Conf. Ser., 213, 167

Jordan, E. C., \& Balmain, K. G. 1985, Electromagnetic Waves and Radiating Systems, ed. W. L. Everitt (Prentice-Hall, Inc.), 636

Kern, P., Malbet, F., Schanen-Duport, I., \& Benech, P. 1997, Integrated optics single-mode interferometric beam combiner for near infrared astronomy (Integrated Optics for Astronomical Interferometry), 195

Kervella, P., Gitton, P. B., Segransan, D., et al. 2003, in Interferometry for Optical Astronomy II, ed. W. A. Traub, Proce. SPIE, 4838, 858

Léger, A., Puget, J.-L., Mariotti, J. M., Rouan, D., \& Schneider, J. 1995, Space Sci. Rev., 74, 163
Laurent, E. 2003, Ph.D. Thesis

Léger, A., Mariotti, J. M., Mennesson, B., et al. 1996, Icarus, 123, 249

Malbet, F., Kern, P., Schanen-Duport, I., et al. 1999, A\&AS, 138, 135

Marcuse, D. 1974, Theory of dielectric optical waveguides (New York, Academic Press, Inc.), 267, 62

Mayor, M., \& Queloz, D. 1995, Nature, 378, 355

Mennesson, B., Ollivier, M., \& Ruilier, C. 2002, Optic. Soc. Am. J. A, 19, 596

Ollivier, M., \& Mariotti, J. 1997, Appl. Opt., 36, 5340

Ordal, M. A., Long, L. L., Bell, R. J., et al. 1983, Appl. Opt., 22, 1099

Quéré, Y. 1988, Physique des Matériaux, Cours de l'École Polytechnique (Ed. Ellipses), 464

Rizzi, P. A. 1988, Microwave Engineering - Passive Circuits (Prentice-Hall)

Schanen, I. 2003, Review and requirements of integrable functions report (ESA Contract No. 16847/02/NL/SFe), 22

Wallner, O., Artjuschenko, V. G., \& Flatscher, R. 2004, in New Frontiers in Stellar Interferometry, Proc. SPIE, 5491, ed. W. A Traub, Bellingham, WA: The International Society for Optical Engineering, 636

Wehmeier, U. J., Swain, M. R., Drouet D'Aubigny, C. Y., Golish, D. R., \& Walker, C. K. 2004, in New Frontiers in Stellar Interferometry, Proc. SPIE, 5491, ed. W. A. Traub, Bellingham, WA: The International Society for Optical Engineering, 1435 